\title{
基于等离子体技术制作微流控纸芯片及其在血糖检测中的应用研究
}

\author{
严春芳余思扬蒋艳何巧红* 陈恒武* \\ (浙江大学化学系 微分析系统研究所 杭州 310058)
}

\begin{abstract}
摘要 滤纸经十八烷基三氯硅烷(OTS)疏水化处理以后，用等离子体区域降解滤纸纤维表面的 OTS 疏水单分子层，使 滤纸的局部区域恢复亲水性, 得到具有亲疏水图案化的微流控纸芯片. 考察了等离子体处理时间对滤纸表面亲水性、 亲水深度(水溶液由滤纸表层下渗至内部的纵向深度)的影响. 优化模具的设计, 依据对滤纸亲水深度的不同需求, 设计 了两种 PMMA-PDMS 复合片的组合模具. 初步探讨了该亲疏水性变化过程的化学机理. 将制得的纸芯片用于人体全血. 中血糖含量的测定，线性范围为 $1.7 \sim 17.7 \mathrm{mmol} \cdot \mathrm{L}^{-1}$, 可满足血液样品中血糖的测定.
\end{abstract}

关键词 纸芯片; 十八烷基三氯硅烷; 等离子体; 全血; 葡萄糖

\section{Fabrication of Paper-based Microfluidic Devices by Plasma Treatment and Its Application in Glucose Determination}

\author{
Yan, Chunfang Yu, Siyang Jiang, Yan He, Qiaohong* Chen, Hengwu* \\ (Department of Chemistry, Institute of Microanalytical Systems, Zhejiang Universtity, Hangzhou 310058)
}

\begin{abstract}
Currently, various methods for fabricating microfluidic paper-based analytical devices ( $\mu$ PADs) have been proposed due to their great potential applications in many fields such as clinical diagnosis, food quality control and environmental monitoring. Hereby, a novel and simple method for the fabrication of microfluidic paper-based analytical devices via plasma treatment is reported. Paper was first hydrophobized via octadecyltrichlorosilane (OTS) silanization. The OTS silanized paper was then region-selectively plasma-treated via a mask with channel network. The plasma-exposed area of the paper was turned to hydrophilic channel network due to the degradation of hydrophobic OTS molecules coupled to the paper's cellulose fibres before. Two types of hybrid polymethylmethacrylate-polydimethylsiloxane (PMMA-PDMS) masks were developed to obtain well defined hydrophilic channel with the required depth. With the elastic PDMS piece adhered to the rigid PMMA piece, it excellently solved the problem of the expansion of hydrophilic channel caused by the leakage of plasma atmosphere in the gap between the mask and paper. The effect of plasma-treating time on hydrophilicity of paper was investigated. The water contact angle (WCA) dramatically decreased from $133.9^{\circ} \pm 1.3^{\circ}$ to $0^{\circ}$ with the prolonging of the plasma treating time from $0 \mathrm{~s}$ to $30 \mathrm{~s}$. Meanwhile, the depth of wettable channel could also increase to nearly the thickness $(180 \mu \mathrm{m})$ of the paper after treated for $30 \mathrm{~s}$. Attenuated total reflectance Fourier transformed infrared (ATR-FT-IR) spectrometer and X-ray photoelectron spectroscopy (XPS) were applied to characterize the surface chemistry of paper during silanization and plasma treatment, and the related mechanism was discussed. The fabricated $\mu \mathrm{PAD}$ was applied for detection of plasma glucose in whole blood. After diluted with $2 \% \mathrm{NaCl}$ by a ratio of $1: 4$, plasma could be separated from blood cells due to their different mobility in the channel on paper. The separated plasma reached test readout zone and reacted with the substrates of Trinder's reaction. The analytical results observed with the $\mu$ PAD-based colorimetric assays agreed well with those determined by conventional glucose meter.
\end{abstract}

Keywords microfluidic paper-based analytical devices; octadecyltrichlorosilane; plasma; whole blood; glucose

\section{1 引言}

微流控纸芯片(microfluidic paper-based chip)又称纸 上微型实验室(lab on paper), 是一种在纸基底上构建亲 疏水的通道网络以实现分析检测的新型微流控分析器 件(microfluidic paper-based analyitical devices, $\mu$ PADs). 自 2007 年 whitesides 组 ${ }^{[1]}$ 首次提出这个概念以来, 纸芯
片因其原料廉价易得、易加工、生物兼容性好、无需外 力驱动液流等特点受到了科研工作者极大的关注, 在临 床医学诊断, 食品安全检测以及环境质量监控等多个领 域展现了巨大的应用前景.

近几年来，文献相继报道了紫外光处理 ${ }^{[1 \sim 4] \text { 、蜡 }}$ 印 $^{[5,6] 、 \text { 溶剂刻蚀 }}{ }^{[7,8]}$ 、等离子体处理 ${ }^{[9]}$ 、绘图 ${ }^{[10 ~ 12]}$ 等多

\footnotetext{
*E-mail: heqh@zju.edu.cn; Tel.: 0086-0571-88206773; Fax: 0086-0571-88273572; hwchen@zju.edu.cn; Tel.: 0086-0571-88206773; Fax: 0086-057188273572

Received June 30, 2014; published August 11, 2014.

Supporting information for this article is available free of charge via the Internet at http://sioc-journal.cn.

Project supported by the National Basic Research Program of China (973 Program, No. 2007CB714502) and the National Natural Science Foundation of China (No. 20890020).

项目受国家重点基础研究发展计划(973 计划, No. 2007CB714502)和国家自然科学基金(No. 20890020)资助.
} 
种微流控纸芯片的制作方法 ${ }^{[13 \sim 15]}$. Whitesides 组 ${ }^{[1]}$ 最早 用 SU-8 光胶涂覆滤纸, 在掩膜保护下紫外光(UV)辐照 特定区域, SU-8 经光聚合在滤纸上形成疏水坝, 形成分 辨率较高的液流通道. 最近, 本课题组 ${ }^{[3]}$ 将十八烷基三 氯硅烷(OTS)通过化学键合, 自组装于滤纸纤维表面, 形成疏水化滤纸(简称 OTS-滤纸), 再利用 $\mathrm{UV} / \mathrm{O}_{3}$ 区域性 降解自组装单分子层, 成功制得高分辨率、耐溶剂且耐 折叠的纸芯片. 也有文献报道利用等离子体处理疏水化 滤纸制备纸芯片. 例如, Whitesides 组 ${ }^{[1]}$ 在用 UV 光刻 SU-8 制作纸芯片的过程中, 引入等离子体氧化步骤, 以 进一步增强其通道的亲水性能. $\mathrm{Li}$ 等 ${ }^{[9]}$ 在滤纸上修饰烷 基烯酮二聚体，利用等离子体处理夹在镂空金属模具中 的滤纸, 得到所需的亲水通道网络.

本文研究建立了一种基于 OTS 自组装疏水化-等离 子体降解 OTS 单分子层的纸芯片加工新方法. 对模具 进行改良和优化, 较好地规避了等离子体气氛的渗漏问 题, 成功制备出具有亲/疏水通道网络的微流控纸芯片, 并将其应用于全血中血糖的检测.

\section{2 结果与讨论}

\section{1 等离子体处理初步试验}

本课题组前期工作中已建立了一套较为完整的基 于紫外光降解自组装单分子层的纸芯片加工方法, 该方 法过程简单易操作, 所用硅烷化试剂廉价易得、种类繁 多, 制得的纸芯片通道分辨率高. 但是因其耗时 $(90$ min)较长, 制作成本相对较高, 且紫外辐照容易造成滤 纸发黄、老化等现象, 对应用造成一定的影响. 本文试 采用等离子体降解滤纸上键合的 OTS 分子层, 初步探 究发现, 在真空等离子体清洗器中处理 $30 \mathrm{~s}$ 后的 OTS滤纸(简称 Plasma-OTS-滤纸), 其表面水的接触角由 $134^{\circ}$ 成功降至 $0^{\circ}$, 证实了等离子体气氛可成功快速降解 纤维素表层的 OTS, 恢复其亲水性. 若辅以适当的模具 即可制得具有亲疏水图案化的纸芯片.

\section{2 等离子体处理时间的选择与优化}

等离子体降解 OTS 自组装层的关键之一就是处理 时间. 如图 1 所示, 随着等离子体降解时间的延长, OTS-滤纸表面的水接触角下降趋势明显, 由处理前的 $134^{\circ}$ 逐步降低, 至 $25 \mathrm{~s}$ 时, 接触角已趋近于 $0^{\circ}$, 滤纸恢 复其原有的亲水性(图 1a 所示). 与此同时, 滤纸纵向的 亲水深度(测量方法见支持信息)也随着处理时间延长而 不断增大(图 1b 所示). 未经等离子体处理时, 水溶液呈 球形附着于疏水的 OTS-滤纸上, 同时由于水滴重力的 作用, 迫使少量水会沿着滤纸纤维孔隙下沉, 实际测得 滤纸纵向被水溶液浸润深度约 $30 \mu \mathrm{m}$. 处理 $15 \mathrm{~s}$ 后, 水 可以在毛细管力作用下, 渗透至将近滤纸一半的厚度 $(86 \mu \mathrm{m})$; 至 $25 \mathrm{~s}$ 时, 溶液的下渗深度已接近整张滤纸的 厚度 $(180 \mu \mathrm{m})$. 因此, 可根据不同要求, 选择合适的处
理时间以获得相应深度的亲水通道.
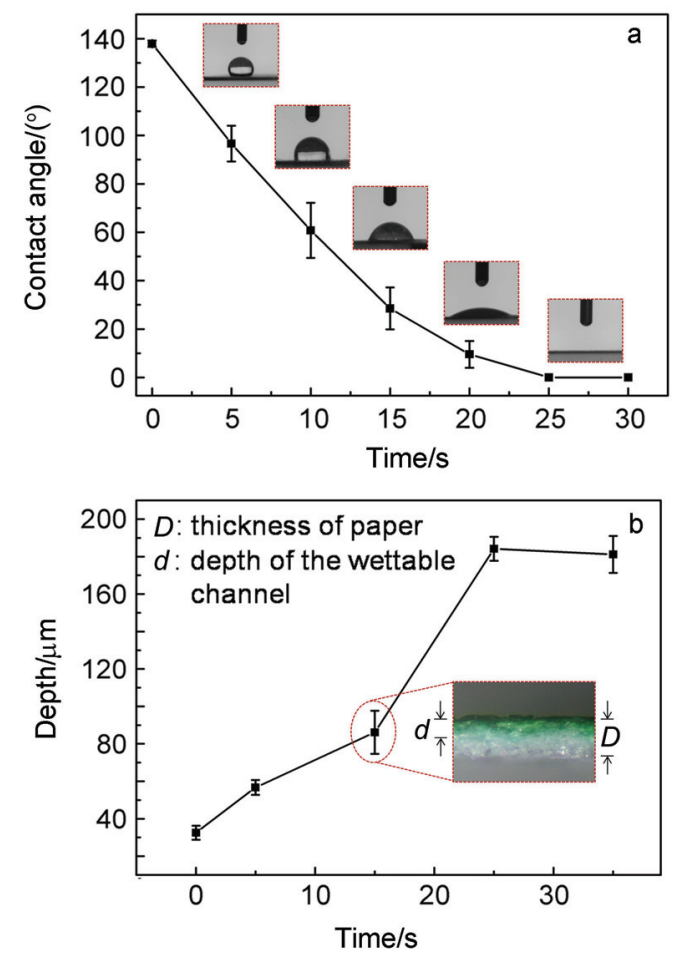

图 1 等离子体处理时间与滤纸亲水程度的关系图 (a) 水的接触角-等离子体处理时间关系图; (b) 亲水通道深度-等离子体 处理时间关系图

Figure 1 Effect of plasma treating time on wettability of filter paper (a) water contact angle against plasma treating time; (b) depth of the hydrophilic channel against plasma treating time

\section{3 模具的设计与优化}

模具的设计是在纸芯片上构建高精度亲/疏水通道 网络的关键. 采用等离子体选择性处理 OTS-滤纸时, 等离子气氛易从模具与滤纸的缝隙处渗漏扩散, 引起亲 水图案的扩张, 影响图案分辨率. 因此, 本实验考察了 多种模具对图案分辨率的影响. 图 $2 \mathrm{a}$ 是设计的花型镂 空模具构型及相应的尺寸, 圆形区域 $\mathrm{R}$ 直径 $3.5 \mathrm{~mm}$, 通 道 C 宽度为 $2.0 \mathrm{~mm}$. 分别将 OTS-滤纸夹心于两片具有 图 $2 \mathrm{a}$ 所示镂空花形图案的 PMMA、铝合金、以及 PMMA-PDMS 复合片(制作方法见实验部分 4.1.2 节)中, 用文具夹夹紧，置于等离子体腔内处理 $30 \mathrm{~s}$ 后，在花型 的中心区域加入 $15 \mu \mathrm{L}$ 绿色食用色素水溶液, 在毛细作 用下溶液沿亲水区域扩散，其效果如图 $2 \mathrm{~b} \sim$ 图 $2 \mathrm{~d}$ 所示. 经测量, 采用 PMMA 和铝合金模具时, 等离子体处理后 亲水区的 $\mathrm{R}$ 直径分别增大至 $4.5 \mathrm{~mm} 、 4.2 \mathrm{~mm}$, 通道 $\mathrm{C}$ 扩宽约 50\%. 可见这两种硬质模具的密封性能欠佳, 不 能完全抵挡等离子体气氛的扩散渗漏; 而采用 PMMA-PDMS 复合片作为模具时, 效果明显改善, 亲水 图案基本没有扩张(图 2d 所示). 由此可见, PDMS 因其 较好的弹性, 可作为密封层, 增强滤纸与模具间的密闭 性, 因此, 本实验选择 PMMA-PDMS 复合片用于模具 的组成. 


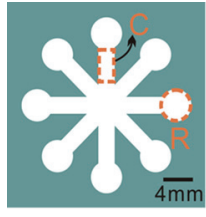

a

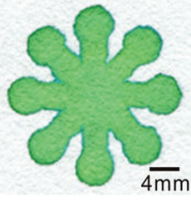

b

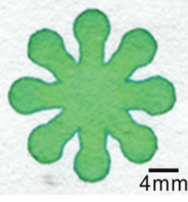

c

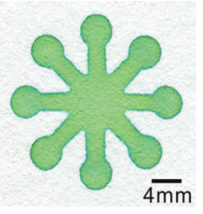

d
图 2 不同模具的图案化效果图

(a) 花型模具图案构型; (b) PMMA 模具的图案化效果; (c) 铝合金模具的 图案化效果; (d) PMMA-PDMS 复合模具的图案化效果

Figure 2 Comparison in the performance of masks used to pattern the OTS-paper via plasma treatment.

(a) original pattern of the masks. Wetted area of the plasma-treated paper masked with (b) a PMMA mask, (c) an aluminum mask, and (d) a hybrid PMMA-PDMS mask

为满足对滤纸亲水深度的不同需求, 本实验设计如 图 3 所示的两种类型模具: $M_{1}$, 上层为具有镂空图案的 PMMA-PDMS 复合片, 下层为平整的 PMMA-PDMS 垫 片, 简称单镂空模具; $\mathrm{M}_{2}$, 上下均为具有镂空图案的 PMMA-PDMS 复合片, 简称双镂空模具. 其中, 模具 $\mathrm{M}_{1}$ 可用于制作亲水通道深度小于滤纸厚度、背面保持 疏水性能的纸芯片, 可用于负载溶液, 作为储液池或反 应区; 模具 $\mathrm{M}_{2}$ 可用于制作亲水通道深度等于滤纸厚度、 水溶液需要渗透穿过整个滤纸纵向深度的微流控纸芯 片，此类纸芯片可用于过滤等分离分析.

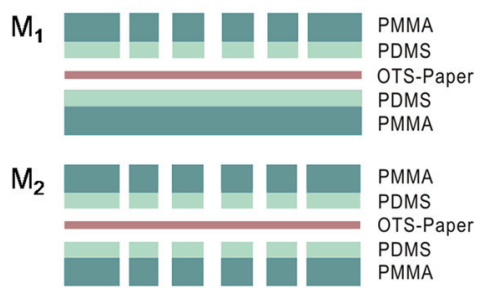

图 3 PMMA-PDMS 复合模具构型图

$M_{1}$, 单镂空模具; $M_{2}$, 双镂空模具

Figure 3 Schematic structure of the hybrid PMMA-PDMS masks $\mathrm{M}_{1}$, mask for single-side patterning; $\mathrm{M}_{2}$, mask for double-side patterning

分别采用上述两种模具对 OTS-滤纸进行不同时间 的等离子体处理, 然后在滤纸花型亲水区域正面的中心 滴加 $15 \mu \mathrm{L}$ 色素水溶液, 滤纸的正面和背面图案化效 果如图 4 所示. 其中, 图 4a 为采用单镂空模具所得的图 案化效果, 可见, 处理时间小于 $20 \mathrm{~s}$ 时, 只有局部区域 亲水，绿色食用色素的水溶液不能完全浸润整个亲水区 域(图案不完整); 当处理时间延长至 $20 \mathrm{~s}$ 后, 滤纸正面 的花形区域已完全亲水, 但背面依然保持一定的疏水 性, 如图 4c 所示, 对于单面处理 $30 \mathrm{~s}$ 的 OTS-滤纸, 在其 背面花形区域中心滴加 $15 \mu \mathrm{L}$ 红色色素溶液后, 液滴依 然可呈球形立于纸上, 接触角维持在 $120^{\circ}$ 左右, 说明滤 纸背面疏水性良好. 而采用双镂空模具时(图 4b 所示), 由于等离子体气氛可从正反面同时对 OTS 进行降解, 处理 $20 \mathrm{~s}$ 后, 溶液即可渗透至背面, 双面图案基本一致, 而且处理时间延长至 $45 \mathrm{~s}$ 时，亲水通道无明显扩张.
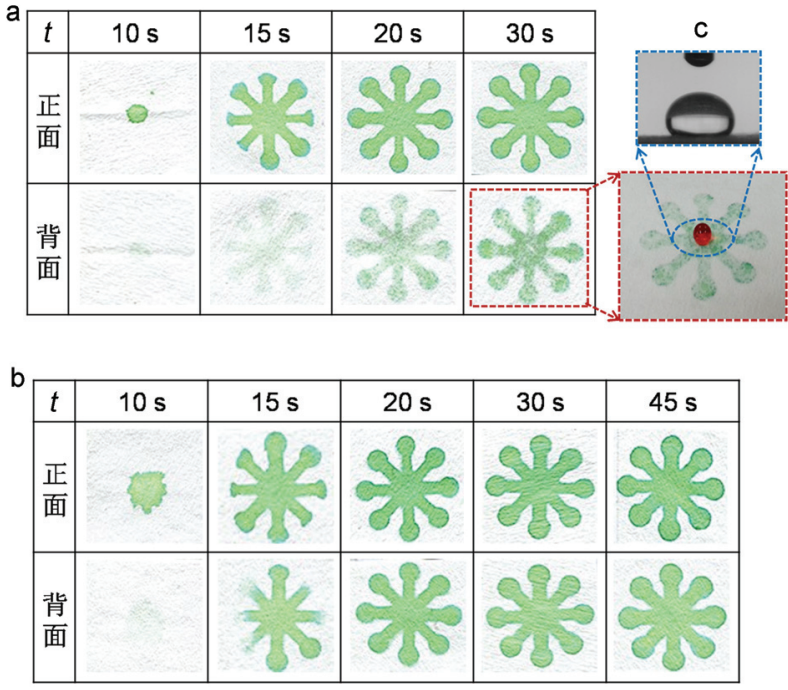

图 4 单面和双面等离子体处理的图案化效果

(a) 利用单镂空模具的图案化效果; (b) 利用双镂空模具的图案化效果; (c) 单面处理 $30 \mathrm{~s}$ 时滤纸背面的水滴

Figure 4 Effect of plasma treating time on the patterning of the OTS-paper via single-side (a) and double-side (b) plasma treatment; (c) a water droplet on the back of OTS-paper after plasma treatment for $30 \mathrm{~s}$

\section{4 机理讨论}

为探讨滤纸处理前后亲水性变化机理, 分别对原滤 纸、OTS-滤纸及 Plasma-OTS-滤纸进行衰减全反射红外 (ATR-FT-IR)与 X 射线光电子能谱(XPS)表征, 结果见图 5 和图 6. 由图 5 可知, OTS-滤纸相较于原滤纸, 在 2916 和 $2853 \mathrm{~cm}^{-1}$ 处新增两个明显的尖峰, 分别对应于 OTS 长链烷烃中- $\mathrm{CH}_{2}$-的不对称和对称伸缩振动峰; 图 6(b) 中新增的 Si2s 和 Si2p 两个硅峰, 也能说明 OTS 已接枝 于滤纸表面. 经等离子体处理后, 长链烷烃的伸缩振动 峰消失, 随之出现 $1708 \mathrm{~cm}^{-1}$ 处的 $\mathrm{C}=\mathrm{O}$ 峰, 推测是 OTS-滤纸表面疏水性的碳链被等离子体降解, 随之产 生含氧活性基团, 从而使滤纸恢复其亲水性, 这与三种 滤纸的 XPS 谱图计算所得的氧/碳含量比变化趋势相一 致. 此外, 与文献[3]相比, 等离子体处理后滤纸纤维素 骨架的四个在 1160、1110、1056、1023 $\mathrm{cm}^{-1}$ 处的红外 特征吸收峰基本保持不变(图 5c 所示), 说明本方法并未 破坏滤纸本身的纤维素骨架结构. 因此, 相较于 $\mathrm{UV} / \mathrm{O}_{3}$ 处理方法, 等离子体处理是一种温和的 OTS 降解方式, 对滤纸本身没有造成破坏，可避免滤纸发黄、变脆等老 化现象.

\section{5 微流控纸芯片在血糖快速检测中的应用}

\subsection{1 全血血糖检测的纸芯片设计思路}

纸芯片上葡萄糖含量的检测方法主要有比色 法 ${ }^{[16,17]}$ 、化学发光法 ${ }^{[18,19]}$ 和电化学法 ${ }^{[20,21]}$ 等. 本文参照 文献[17], 利用 Trinder 反应所得显色产物的颜色深浅对 血液中葡萄糖含量进行测定. 


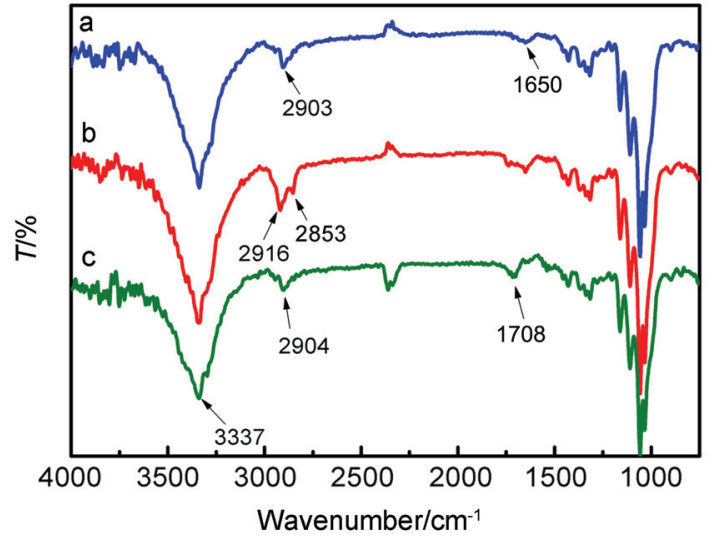

图 5 滤纸的衰减全反射红外光谱

(a) 原滤纸; (b) OTS-滤纸; (c) Plasma-OTS-滤纸

Figure 5 ATR-FT-IR spectra for paper at different stage of surface modification

(a) native paper; (b) OTS-paper; (c) Plasma-OTS-paper

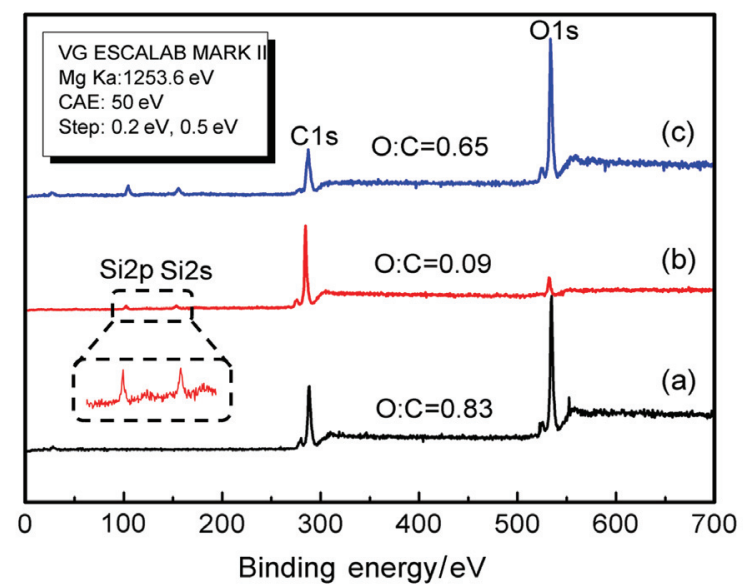

图 6 滤纸的 X 射线光电子能谱

(a) 原滤纸; (b) OTS-滤纸; (c) Plasma-OTS-滤纸

Figure 6 XPS spectra for paper at different stage of surface modification

(a) native paper; (b) OTS-paper; (c) Plasma-OTS-paper

在常规的血液分析中, 多数需要对样品进行离心、 沉降等处理，将血浆或血清从全血中分离出来进行检 测, 以避免血细胞对其造成干扰. 在纸芯片上实现全血. 中目标物的测定, 关键在于如何将血细胞的分离过程与 血浆中标志物的检测过程集成于同一芯片装置. Vella 等 ${ }^{[22]}$ 将血液分离膜与图案化滤纸进行堆叠, 构建三维 纸芯片, 通过中间层滤膜的过滤作用, 实现血细胞与血 浆的分离, 在下层滤纸的反应区实现了血浆中碱性磷酸 酶(ALP)等物质的检测. Songjaroen 等 ${ }^{[23]}$ 将分离膜与滤 纸平行组装, 血液沿通道渗透时, 血细胞被分离膜阻拦 捕集，而血浆则扩散至滤纸的显色区. 此外，Yang 等 ${ }^{[24]}$ 仅依靠滤纸, 通过外加抗体促使血细胞凝集, 也成功实 现了全血样品中葡萄糖含量的测定. 本文设计如图 7 所 示通道构型, $S$ 为血液进样区, $B$ 作为缓冲区域, 减弱血 细胞的流动速度, $\mathrm{R}$ 作为显色区域, 预先负载酶底物和
显色底物. 并利用适宜浓度的 $\mathrm{NaCl}$ 溶液作为血液的稀 释剂, 尝试利用血细胞和血浆在纤维通道内流动性的差 异，将两者分离. 利用不同浓度的 $\mathrm{NaCl}$ 溶液 $(0.9 \%$ 、 $2.0 \% 、 3.0 \% 、 4.0 \%)$ 稀释全血，血细胞和血浆的分离效 果如图 $8 \mathrm{a}$ 所示. $0.9 \%$ 的 $\mathrm{NaCl}$ 溶液不足以使血浆从全血. 中分离, $3 \%$ 和 $4 \%$ 的 $\mathrm{NaCl}$ 溶液虽然可以加剧血细胞凝 固, 但是血浆的流动性也大大降低[图 8(3 4)所示]. 因 此, 本实验采用浓度为 $2 \%$ 的 $\mathrm{NaCl}$ 溶液作为稀释剂, 适 当促进蛋白凝结，降低血细胞的流动性，分离效果较好. 同时，考察不同稀释比例对分离效果的影响，结果如图 $8 \mathrm{~b}$ 所示. 稀释比例较低时, 血液样品粘稠度较大, 流动 性较差, 不利于后续分析. 故本文选取 $2 \%$ 的 $\mathrm{NaCl}$ 溶液, 以 $1: 4$ 的配比稀释全血, 使细胞与血浆在滤纸通道内 侧向流动的过程中自然分离.

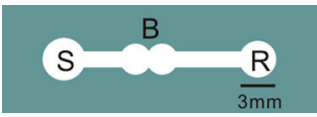

图 7 用于血糖检测的纸芯片通道构型图 $S$, 进样区; B, 缓冲区域; $R$, 显色区域

Figure 7 Layout of the paper-based microfluidics used for determination of glucose in human blood

$\mathrm{S}$, sample dosing site; B, buffering area; $\mathrm{R}$, test readout zone

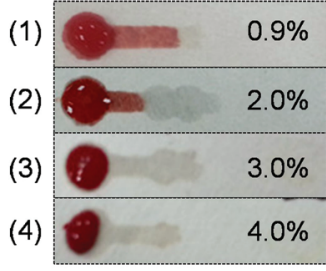

(a)
(5)

(6)

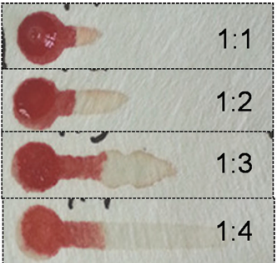

(b)
图 8 稀释剂中 $\mathrm{NaCl}$ 浓度(a)以及试样稀释比例(b)对血细胞与血浆在 芯片上分离效果的影响

经稀释剂稀释后的全血试样滴加在左侧进样区内, 血细胞和血浆在毛细 作用力驱使下, 沿亲水通道向右侧渗透扩散. 红色前沿指示血细胞所达之 处, 褐灰色前沿指示血浆所达之处

Figure 8 Effects of $\mathrm{NaCl}$ concentration in diluent (a) and sample dilution ratio (b) on the efficiency of on-chip separation of blood cells from plasma

After diluted blood sample was pipetted into the left sample reservoir, blood cells and plasma solution moved towards right in the hydrophilic channel with different velocities. The red front indicates where blood cells reached while the brown-grey front indicates where the plasma reached

\subsection{2 纸芯片上全血样品葡萄糖的检测}

根据 4.2 节所示方法配制以真实血样为基底的葡萄 糖标准溶液. 平行测定三组, 将显色结果扫描成像, 转 化为灰度即可得标准曲线. 如图 9 所示，血糖浓度在 1.7 $17.7 \mathrm{mmol} \cdot \mathrm{L}^{-1}$ 之间时，平均灰度与浓度间的线性 回归方程为 $y=1.9358 x+74.457$ (式中 $x$ 为折算成稀释前 的血液中葡萄糖浓度, 单位为 $\mathrm{mmol} \cdot \mathrm{L}^{-1}, y$ 表示灰度值, 无量纲), 线性相关系数 $R^{2}$ 为 0.9881 . 分别采集含低、 中、高血糖值的血液样品, 由 $2 \%$ 的 $\mathrm{NaCl}$ 溶液稀释 4 倍 即得待测样品液. 将测定结果的灰度值代入回归方程, 计算所得样品中血糖值列于表 1 , 并与快速血糖仪 
(Accu-Chek Active 罗康全活力型)的测定值相比较, 结 果基本一致.

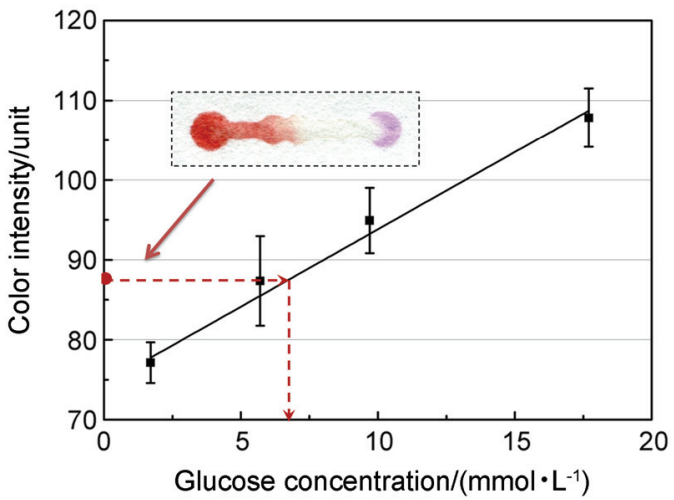

图 9 纸芯片上全血样品中血糖测定结果

Figure 9 Quantification of glucose concentration in whole blood sample by using the $\mu$ PADs $(n=3)$

表 1 全血的血糖测定结果

Table 1 The determination of glucose in whole blood sample

\begin{tabular}{cccccc}
\hline & \multicolumn{2}{c}{ Proposed method } & & \multicolumn{2}{c}{ Reference method $^{a}$} \\
\cline { 2 - 3 } \cline { 5 - 6 } & $\begin{array}{c}\mathrm{mmol} \cdot \mathrm{L}^{-1} \\
(\text { Mean } \pm \mathrm{SD}, n=3)\end{array}$ & $\mathrm{RSD} / \%$ & & $\begin{array}{c}\mathrm{mmol} \bullet \mathrm{L}^{-1} \\
(\text { Mean } \pm \mathrm{SD}, n=3)\end{array}$ & $\mathrm{RSD} / \%$ \\
\hline Sample 1 & $3.6 \pm 0.5$ & 13.9 & & $3.1 \pm 0.1$ & 3.2 \\
Sample 2 & $6.6 \pm 0.8$ & 12.1 & & $6.3 \pm 0.1$ & 1.6 \\
Sample 3 & $11.1 \pm 1.2$ & 10.8 & & $10.6 \pm 0.2$ & 1.9 \\
\hline
\end{tabular}

${ }^{a}$ Determined by Accu-Chek Active Blood Glucose Meter (Roche Diagnostics $\mathrm{GmbH}, \mathrm{GER})$.

\section{3 结论}

本文建立了一种通过等离子体区域选择性降解滤 纸表面 OTS 疏水化层的纸芯片制作新方法. 采用 PMMA-PDMS 复合模具, 能有效避免等离子体气氛渗 漏所造成的亲水通道扩张, 实现模具图形向滤纸的高精 度转移. 利用血. 细胞与血浆在芯片通道内流动性的差 异, 实现两者的分离, 确保显色反应不受血细胞的干扰. 本方法加工速度快，与环境友好，成本低廉，利于推广。 制得的纸芯片可供非专业人员用于全血中血糖含量的 快速测定.

\section{4 实验部分}

\section{1 纸芯片的制作过程}

\subsection{1 滤纸硅烷化处理}

根据文献[3]对滤纸进行硅烷化处理: 将滤纸浸于 含 $0.1 \%(V / V)$ OTS 的正已烷溶液, $5 \mathrm{~min}$ 后取出, 依次用 正已烷和水漂洗, $\mathrm{N}_{2}$ 吹干, 即可得到疏水的 OTS-滤纸, 室温下密封保存(可稳定半年以上), 供后续实验使用.

\subsection{2 模具的制作}

采用 ArtCAM 2008 软件设计图案, 利用阿莫雕刻 机(东莞市东城阿莫电子经营部, 广东)在厚度 $2 \mathrm{~mm}$ 的 PMMA 板材上刻出所需图形. 根据实际需要调整雕刻 深度, 分别制作出具有镂空图案的 a 片(雕刻深度 $2 \mathrm{~mm}$ ) 与非镂空图案的 b 片(雕刻深度 $1 \mathrm{~mm}$ ). 如图 10 所示, a 片与 $\mathrm{b}$ 片构成一组图案相反的阴阳模, 其中前者直接用 于组成等离子体处理时所需模具, 镂空部分即可透过等 离子体气氛, 在 OTS-滤纸中形成亲水区域; 后者作为 阳模, 用于浇注厚度略小于 $1 \mathrm{~mm}$ 的 PDMS 片, 将其与 a 片贴合，即可得镂空的 PMMA-PDMS 复合片 A. 采用平 板 (不带图案) PMMA 和 PDMS 片组合可得 PMMA-PDMS 垫片 $\mathrm{B}$. 等离子体处理时，根据亲水通道 (区域)是否需要贯穿整片滤纸, 可分别选用单镂空模具 $(A+B)$ 或双镂空模具 $(A+A)$.

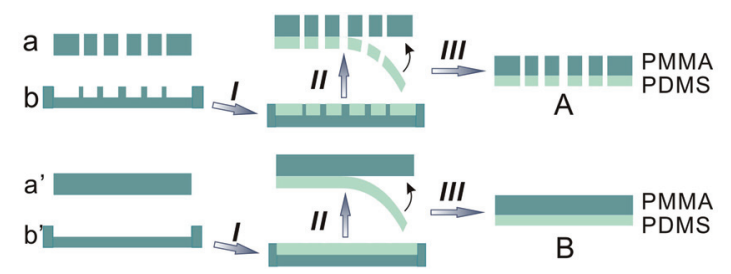

图 10 PMMA-PDMS 复合片制作流程图

I. 浇注 PDMS 于 $\mathrm{b}$ 片; II. 将 PDMS 剥离, 并与 $\mathrm{a}$ 贴合; III. 形成 PMMA-PDMS 复合片

Figure 10 Schematic of the process for the fabrication of PMMA-PDMS hybrid pieces

I. pour PDMS on the piece of $b$ and cure it; II. remove PDMS from the piece of $b$ and adhere to piece of a; III. formation of hybrid PMMA-PDMS piece

\subsubsection{OTS-滤纸的区域选择性等离子体处理}

将硅烷化滤纸夹心于 PMMA-PDMS 复合模具中, 用文具夹夹紧固定，置于等离子体清洗器的腔体中央， 功率设为高档，在真空条件下处理 $30 \mathrm{~s}$, 取出，依次用 正已烷和水漂洗，氮气吹干，即可在疏水的硅烷化滤纸 表面形成亲水通道网络. 完整的纸芯片制作流程如图 11 所示.

\section{2 血糖检测}

葡萄糖标准溶液的配制: 首先以 $2 \% \mathrm{NaCl}$ 水溶液为 溶剂，配制不同浓度的葡萄糖溶液 $(0.0 、 1.0 、 2.0 、 4.0$ $\left.\mathrm{mmol} \cdot \mathrm{L}^{-1}\right)$, 再分别将其与某已知血糖浓度的人体全血 按 4:1(V/V)混合, 即可得以真实血液作为基底的葡萄 糖标准溶液.

待测血液样品溶液的配制：以 $2 \% \mathrm{NaCl}$ 水溶液为溶 剂, 将待测的人体全血释成 $20 \%(V / V)$ 血液样品.

检测时, 首先滴加 $0.4 \mu \mathrm{L}$ 酶底物 $(150 \mathrm{U} / \mathrm{mL}$ GOD, $7.5 \mathrm{U} / \mathrm{mL} \mathrm{HRP}$ )于 $\mathrm{R}$ 区域, 静置 $5 \mathrm{~min}$ 后, 继续滴加 0.4 $\mu \mathrm{L}$ 显色底物 $\left[0.3 \mathrm{~mol} \cdot \mathrm{L}^{-1}\right.$ 海藻糖, $2.2 \mathrm{mmol} \cdot \mathrm{L}^{-1} 4$-氨基 安替比林(4-AAP), $2.2 \mathrm{mmol} \cdot \mathrm{L}^{-1} \mathrm{~N}$-乙基- $N$-(3-磺丙 基)-3-甲基苯胺钠盐(TOPS)], 避光静置 $5 \mathrm{~min}$. 取 $6 \mu \mathrm{L}$ 
葡萄糖标准溶液至 $\mathrm{S}$ 区域，避光密闭静置 $30 \mathrm{~min}$ 后，由 EPSON 扫描仪扫描成像, 通过 Adobe Photoshop 进行数 据处理, 读取 $\mathrm{R}$ 区域灰度平均值, 绘制标准曲线. 按照 同样方法测得待测血液样品溶液的灰度值, 并根据标准 曲线计算得出其血糖含量.

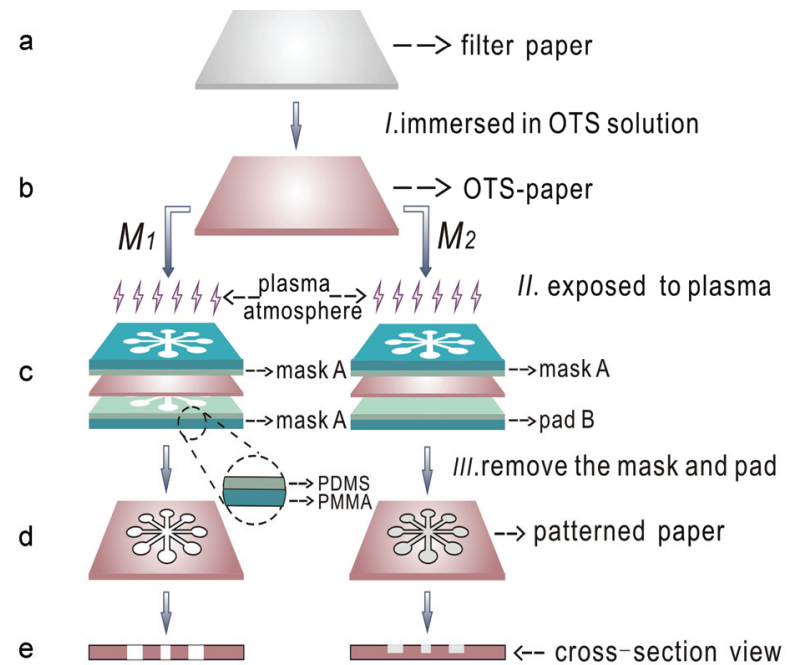

图 11 基于等离子体降解 OTS 单分子层制作纸芯片的流程图 I. 滤纸硅烷化处理; II. 将硅烷化滤纸夹心于 PMMA-PDMS 复合模具中, 等离子体处理; III. 移去模具得到具有亲/疏水通道的纸芯片

Figure 11 Schematic illustration of the process to fabricate paper-based microfluidics via plasma treatment

I. filter paper was silanized; II. OTS-paper was sandwiched between PMMA-PDMS masks and exposed to plasma; III. the masks were removed and a patterned paper was achieved

\section{References}

[1] Martinez, A. W.; Phillips, S. T.; Butte, M. J.; Whitesides, G. M. Angew. Chem., Int. Ed. 2007, 46, 1318.
[2] Martinez, A. W.; Phillips, S. T.; Wiley, B. J.; Gupta, M.; Whitesides, G. M. Lab Chip 2008, 8, 2146.

[3] He, Q. H.; Ma, C. C.; Hu, X. Q.; Chen, H. W. Anal. Chem. 2013, 85 , 1327.

[4] Bai, P.; Luo, Y.; Li, Y.; Yu, X. D.; Chen, H. Y. Chin. J. Anal. Chem. 2013，41，20. (白鹏，罗雁，李英，余晓东，陈洪渊，分析化学， 2013, 41, 20.)

[5] Carrilho, E.; Martinez, A. W.; Whitesides, G. M. Anal. Chem. 2009 , 81,7091 .

[6] Lu, Y.; Shi, W. W.; Qin, J. H.; Lin, B. C. Anal. Chem. 2010, 82, 329.

[7] Abe, K.; Suzuki, K.; Citterio, D. Anal. Chem. 2008, 80, 6928.

[8] Abe, K.; Kotera, K.; Suzuki, K.; Citterio, D. Anal. Bioanal. Chem. 2010, 398, 885

[9] Li, X.; Tian, J. F.; Nguyen, T.; Shen, W. Anal. Chem. 2008, 80, 9131.

[10] Bruzewicz, D. A.; Reches, M.; Whitesides, G. M. Anal. Chem. 2008, $80,3387$.

[11] Nie, J. F.; Zhang, Y.; Lin, L. W.; Zhou, C. B.; Li, S. H.; Zhang, L. M.; Li, J. P. Anal. Chem. 2012, 84, 6331.

[12] Zhao, L. C.; Yan, H. T. Acta Chim. Sinica 2012, 70, 1104. (赵联朝, 间宏涛, 化学学报, 2012, 70, 1104.)

[13] Li, X.; Ballerini, D. R.; Shen, W. Biomicrofluidics 2012, 6, 011301.

[14] Liana, D. D.; Raguse, B.; Gooding, J. J.; Chow, E. Sensors 2012, 12, 11505.

[15] Jiang, Y.; Ma, C. C.; Hu, X. Q.; He, Q. H. Prog. Chem. 2014, 26 167. (蒋艳, 马翠翠, 胡贤巧, 何巧红, 化学进展, 2014, 26, 167.)

[16] Martinez, A. W.; Phillips, S. T.; Carrilho, E.; Thomas, S. W.; Sindi, H.; Whitesides, G. M. Anal. Chem. 2008, 80, 3699.

[17] Chen, X.; Chen, J.; Wang, F. B.; Xiang, X.; Luo, M.; Ji, X. H.; He, Z. K. Biosens. Bioelectron. 2012, 35, 363.

[18] Yu, J. H.; Ge, L.; Huang, J. D.; Wang, S. M.; Ge, S. G. Lab Chip 2011, 11, 1286.

[19] Wang, F. F.; Chen, J.; He, Z. K. Chin. J. Anal. Sci. 2011, 11, 120. (王 芳芳, 陈锦, 何治柯, 分析科学学报, 2011, 11, 120.)

[20] Dungchai, W.; Chailapakul, O.; Henry, C. S. Anal. Chem. 2009, 81, 5821

[21] Nie, Z. H.; Nijhuis, C. A.; Gong, J. L.; Chen, X.; Kumachev, A.; Martinez, A. W.; Narovlyansky, M.; Whitesides, G. M. Lab Chip 2010, 10, 477.

[22] Vella, S. J.; Beattie, P.; Cademartiri, R.; Laromaine, A.; Martinez, A. W.; Phillips, S. T.; Mirica, K. A.; Whitesides, G. M. Anal. Chem. 2012, 84, 2883.

[23] Songjaroen, T.; Dungchai, W.; Chailapakul, O.; Henry, C. S.; Laiwattanapaisal, W. Lab Chip 2012, 12, 3392.

[24] Yang, X. X.; Forouzan, O.; Brown, T. P.; Shevkoplyas, S. S. Lab Chip 2012, 12, 274 